\title{
Mechanism Study and the Role of Coupled Surface Plasmon Polaritons in Optical Out-Coupling Enhanced Top-Emitting OLEDs with a Dielectric Capped Ag Cathode
}

\author{
Xue-Liang Wang \\ Soochow University \\ min qian ( $\square$ qianmin@suda.edu.cn ) \\ Soochow University https://orcid.org/0000-0002-9705-7294 \\ Dong-Ying Zhou \\ Soochow University
}

\section{Research Article}

Keywords: Top-emitting OLED, Transparent Ag cathode, capping layer, Surface plasmon polaritons, Microcavity effect, Mechanism study

Posted Date: February 9th, 2022

DOI: https://doi.org/10.21203/rs.3.rs-1319994/v1

License: (c) (1) This work is licensed under a Creative Commons Attribution 4.0 International License.

Read Full License 


\title{
Mechanism Study and the Role of Coupled Surface Plasmon Polaritons in Optical Out-Coupling Enhanced Top-Emitting OLEDs with a Dielectric Capped Ag Cathode
}

\author{
Xue-Liang Wang ${ }^{1,3} \cdot$ Min Qian ${ }^{1,2,{ }^{*}} \cdot$ Dong-Ying Zhou ${ }^{4,{ }^{*}}$
}

${ }^{1}$ Department of Electronic Science and Technology, Soochow University, Suzhou, Jiangsu 215006, China

${ }^{2}$ City University of Suzhou, Suzhou, Jiangsu 215104, China

${ }^{3}$ Advanced Semiconductor Manufacturing Co. Ltd., Shanghai 200233, China

${ }^{4}$ College of Energy, Soochow University, Suzhou, Jiangsu 215006, China

*Min Qian: qianmin@ suda.edu.cn, ${ }^{*}$ Dong-Ying Zhou: dyzhou@ suda.edu.cn

\begin{abstract}
Classical optical transfer matrix method and micro-cavity theory is applied to compute optical out-coupling of Top-Emitting OLEDs (TEOLEDs). Optical paths of various functional organic films and electrode structures are included in the calculations. Fluorescent Alq 3 green TEOLEDs were fabricated and tested based on theoretical calculations. For TEOLEDs with Ag cathodes plus NPB coatings, we experimentally observed significant enhancements in optical out-couplings compared to that of with bare Ag cathode. We also found that thin-film optics theory is insufficient to explain the observed enhancement. To explain the experimental results, we incorporated near-field radiation of electric dipole and its interactions with $\mathrm{Ag}$ cathode film, i.e., surface plasmon polaritons (SPPs) theory, coupled SPPs in a dielectric/metal/dielectric (D/M/D) structure. Therefore, we demonstrate that optical out-coupling enhancement has three contributing factors in a TEOLED: an increased transmittance of cathode Ag, a varied internal micro-cavity gain, and the dominant improved SPP coupling on both sides of the Ag film cathode.
\end{abstract}

Keywords Top-emitting OLED $\cdot$ Transparent Ag cathode•capping layer $\cdot$ Surface plasmon polaritons $\cdot$ Micro-cavity effect $\cdot$ Mechanism study

\section{Introduction}

In the field of OLED display application, most manufacturing companies have made a transition to TEOLED technology, due to the nearly $100 \%$ aperture rate of TFT (Thin Film Transistor) driven AMOLED (Active Matrix OLED). TEOLEDs [1-6] can be fabricated on any solid substrates like silicon wafers or glasses. A TEOLED is constructed using a semi-transparent top cathode such as ITO or Ag thin film and a reflective bottom metal anode. ITO is a very good transparent anode material. It may be used as cathode. ITO's work function, however, is fairly high which does not suit electron injection, decreasing the performance of the device. Other problematic issues in using ITO include indium, which is a very scarce and expensive element, the use of sputtering that damages the organic functional layer, and brittleness which cannot be used in flexible devices[7, 8]. Hence, it is important to explore alternative cathode. Ag is often used as a replacement of ITO for its relatively high optical transparency and good ductility.

In general, a dielectric with high refractive index is deposited on the surface of the metal cathode to promote the out-coupling efficiency. It has been reported that the light extracting efficiency will be increased if an $\mathrm{Ag}$ cathode is coated by index matching materials such as inorganic $\mathrm{ZnSe}, \mathrm{ZnS}, \mathrm{MgF}_{2}, \mathrm{SiO}_{2}, \mathrm{ITO}, \mathrm{ZnO}$, $\mathrm{TiO}_{2}$, organic Alq 3 , NPB, BCP and so on[9-16]. The degree of enhancement varies widely with different types of material and thicknesses.

Some researchers did the transmittance measurement for cathode with different thicknesses of a capping layer, and found that, despite the transmittance through the cathode being at the maximum value, the total device light extracting efficiency is far from the optimal value and sometimes just the opposite[12]. Leo, et al showed that this efficiency enhancement was mainly due to low absorption and high reflection, which leaded to a high extraction because of the micro-cavity effect[13]. Another paper argued that the enhancement of the efficiency is closely related to suppression of surface plasmon polaritons (SPPs), but these authors did not elaborate on the working physics[14]. It is therefore very important to identify various contributing factors in optical out-coupling.

In this paper, using a combination of classic optics and SPP theory, we study the various possible contributing factors on 
enhancing optical out-coupling efficiency in a TEOLED.

\section{Methods}

\section{Metal induced transmission (MIT)}

The metal film is usually used as the transparent cathode in TEOLED and its transparency is the core discussed issue. Many works had been done about the metal cathode's fabrication and properties discussion via experimental measurement. A prominent work is to deposit an inorganic or organic dielectric capping layer on the outgoing surface of the metal cathode and the transmittance was proved to be enhanced efficiently $[17,18]$. The capping layer is sometimes called as refractive index matching layer and the structure is called as the DMD (dielectric/metal/dielectric) structure. But the deep mechanism which often puzzles the researchers is scarcely done, it is often determined with empirical experimental parameters for the metal selection, transparency mechanism and thickness selection without theoretical calculation in many previous literatures $[12,14]$.

In fact, in the thin film optics and optical system research, the metal film is widely used in the design of optical filter. This is just about the issue of metal induced transmission filter (MITF)[19]. From the key knowledge of this point, that is, the absorption and transmittance of a metal film is not only decided by the metal's refractive index $n$, extinction coefficient $k$ and thickness $d$, but also decided by the optical impendence of the adjoining dielectric. As far as the design method of MITF, there is a mature method in thin film optics such as the pattern like this,

$\rightarrow \mid \lambda / 4$ films $\mid$ metal film $\mid \lambda / 4$ films $\mid \rightarrow$

Here, $\lambda / 4$ films refers to anti-reflection films. In this paper, we would not apply this method due to the different case of TEOLED and MITF. The above $\lambda / 4$ anti-reflection films in MITF are hardly to realize in TEOLED fabrication, which is corresponding to the electron injection and transmission layer of cathode. On one hand, the refractive index of organic electronic materials are relative fixed and not easy to regulate to meet the optical path demand; on the other hand, complex films fabrication will add the difficulties and cost to the OLED. Relative speaking, the design of capping layer on the outgoing surface of a metal cathode is pretty easy and meaningful, which is only involved in dielectric selection and thickness optimization.

We explain the enhanced transmission principle via optical transfer matrix method in thin film optics theory and verify it through real TEOLD fabrication. This calculation method is related to metal's dielectric function, complex refractive index, transmittance, reflectance, and so on, and will be briefly discussed as follows.

The energy reflectance $R$, transmittance $T$, absorption $A$ and reflecting phase shift $\varphi$ of equivalent interface can be expressed as ${ }^{10}$

$$
\begin{aligned}
& R=\left|\frac{\eta_{0}-Y}{\eta_{0}+Y}\right|^{2}=\left(\frac{\eta_{0} \cdot B-C}{\eta_{0} \cdot B+C}\right) \cdot\left(\frac{\eta_{0} \cdot B-C}{\eta_{0} \cdot B+C}\right)^{*} \\
& T=\frac{4 \eta_{0} \cdot \eta_{k+1}}{\left(\eta_{0} \cdot B+C\right) \cdot\left(\eta_{0} \cdot B+C\right)^{*}} \\
& A=1-T-R \\
& \varphi=\operatorname{arctg}\left[\frac{i \eta_{0} \cdot\left(C \cdot B^{*}-B \cdot C^{*}\right)}{\eta_{0}{ }^{2} \cdot B \cdot B^{*}-C \cdot C^{*}}\right]
\end{aligned}
$$

Where $Y=C / B, B, C$ can be obtained by optical transfer matrix calculations as follows:

$$
\left[\begin{array}{l}
B \\
C
\end{array}\right]=\prod_{j=1}^{k}\left[\begin{array}{cc}
\cos \delta_{j} & -\frac{i}{\eta_{j}} \sin \delta_{j} \\
-i \eta_{j} \sin \delta_{j} & \cos \delta_{j}
\end{array}\right] \cdot\left[\begin{array}{c}
1 \\
\eta_{k+1}
\end{array}\right]
$$

$\eta_{0}, \eta_{\mathrm{j}}, \eta_{\mathrm{k}+1}$ are the optical admittance of incidence dielectric, the $j$ layer and emergence dielectric, respectively. For convenience, we only discuss the normal incidence and emergence because the optical admittance is reduced to the union form of refractive index for the $s$ and $p$ polarization wave. $\delta_{j}=2 \pi N_{j} d_{j} / \lambda$, the effective phase thickness of the $j$ layer of dielectric, and $N_{j} d_{j}$, the optical thickness.

The refractive index of the metal is obtained by fitting the experimental data from the handbook of solid optical constant of Edward D. Palik's. ${ }^{15}$ The relative dielectric function of metal can be described as ${ }^{16}$

$$
\varepsilon_{r}(\omega)=1-\frac{f_{0} \omega_{p}^{2}}{\omega^{2}+i \gamma_{0} \omega}+\omega_{p}^{2} \sum_{j=1}^{K} \frac{f_{j}}{\omega_{j}^{2}-\omega^{2}-i \gamma_{j} \omega}
$$

where, $\omega_{\mathrm{p}}$ the bulk plasma frequency of a sort of metal, $\gamma_{0}$ the damping coefficient of free electrons, $f_{0}$ the ratio of free electrons in an atom, $\omega_{j}$ the intrinsic frequency of bound electrons, $\gamma_{j}$ the damping coefficient, $f_{j}$ the ratio of bound electrons, $K$ the total kinds number of bound electrons.

The refractive index is the square root of dielectric constant and it is also a complex number for metals.

We use the MATLAB language software to do the simulations and provide the theoretical basis for the metal type and thickness selection[20, 21]. The result is, as a transparent or semi-transparent electrode, $\mathrm{Ag}$ thin film is better than $\mathrm{Al}$ film.

\section{Micro-cavity effect}

The difference between a TEOLED and a normal bottom-emitting 
OLED is its Fabry-Pérot optical resonator micro- cavity effect arising from the high reflectance of the two metal electrodes. The optical resonator can change the light field distribution inside the device, a photon's life time and an emitter's luminescence efficiency[2]. The cavity diagram is provided in Fig. 1, where $T_{2}$ is the transmittance of top electrode, $A_{2}$ the absorbance of cathode, $R_{1}$ and $R_{2}$ the reflectance of the two electrodes, $I_{0}$ and $E_{0}$ the luminescence intensity and electric field of the dipole radiation, $I_{2}$ and $E_{2}$ the emergent light, $L_{1}$ the optical
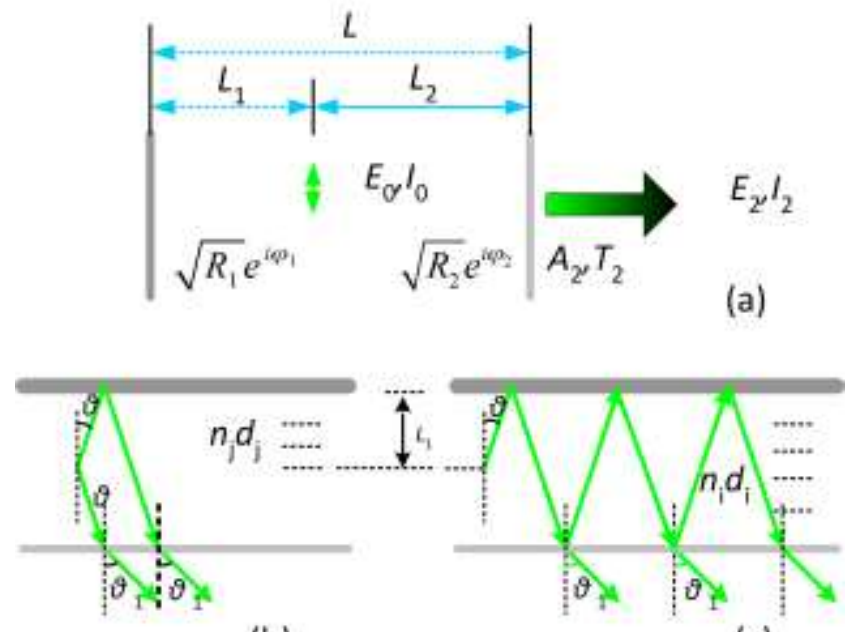

(b)

(c)

Fig. 1. (a) Optical micro-cavity ( $\mathrm{M}_{1}$ : anode; $\mathrm{M}_{2}$ : cathode); (b) wide-angle interference; (c) multi-beam interference

path length from the dipole to the reflective anode, $L_{2}$ the optical path from the dipole to the reflective cathode, $L$ the total micro-cavity length. The emergent light intensity from the semi-transparent cathode at normal direction can be expressed as[20]

$$
\begin{aligned}
& I(\lambda)=\frac{\left.T_{2} \mathrm{~g} 1+R_{1}+2 \sqrt{R_{1}} \cos \left(\frac{4 \pi L_{1}}{\lambda}-\varphi_{1}\right)\right]}{1+R_{1} R_{2}-2 \sqrt{R_{1} R_{2}} \cos \left(\frac{4 \pi L}{\lambda}-\varphi_{1}-\varphi_{2}\right)} I_{0}=G(\lambda) g_{0}=T_{2} \mathrm{~g} F(\lambda) g_{0} \\
& L=L_{1}+L_{2}=\sum n_{i} d_{i}, L_{1}=\sum n_{j} d_{j}, \varphi_{1}=\varphi_{1}(\lambda), \varphi_{2}=\varphi_{2}(\lambda)
\end{aligned}
$$

In equation (1), the numerator represents the wide-angle interference, and the denominator represents the multi-beam interference. Where, $G(\lambda)$ is called as the gain factor, $F(\lambda)$ micro-cavity factor, $n_{i} / d_{i}$ the refractive index and thickness of the $i$ layer of organic material in the micro-cavity, $n_{j} / d_{j}$ those close to the bottom anode, $\lambda$ wavelength of the emergent light, $\varphi_{1}(\lambda) / \varphi_{2}(\lambda)$ inside reflecting phase shift of the two electrodes. From equation (1), when constructive interference occurs, the emergent light reaches to the maximum. It should meet the corresponding conditions:

$$
\begin{aligned}
& \varphi_{1}(\lambda)+\varphi_{2}(\lambda)-\frac{4 \pi}{\lambda} \bullet \sum n_{i} d_{i}=m \bullet 2 \pi \\
& \varphi_{1}(\lambda)-\frac{4 \pi}{\lambda} \bullet \sum n_{j} d_{j}=m^{\prime} \bullet 2 \pi
\end{aligned}
$$

$m, m^{\prime}=0, \pm 1, \pm 2 \ldots$, Here $\lambda$ is the resonant wavelength. Equation (2) represents a constructive multi-beam interference of the total cavity, which determines the thickness of the micro-cavity. Equation (3) indicates the wide-angle constructive interference of the radiation dipole in the cavity, which is determined by the position of the dipole from the two electrodes. We use $\varphi_{1}, \varphi_{2}$ to replace the value of equation $(2,3)$ and take $m=0$ to decide the cavity length parameters of a TEOLED. The change of dielectric thickness of the cavity is selective for the wavelength of the emergent light.

The parameters of light path can be computed via optical film transfer matrix method[22-24]. We perform simulations through the use of MATLAB[25]. The simulated anode structure is glass/ $\mathrm{Al}(100 \mathrm{~nm}) /$ organic, and the dielectric constant of the organic layer is 1.7 . The optical reflectance $R$ is $87 \%$ at $\lambda=528 \mathrm{~nm}$ (the peak wavelength of bottom emitting green OLED of $\mathrm{Alq}_{3}$ ). The calculated reflecting phase shift is $\varphi_{1}=2.6169 \mathrm{rad}$. The cathode structure is organic/Ag $20 \mathrm{~nm}[20,21]$, the reflecting phase shift is $\varphi_{2}=1.7995 \mathrm{rad}$. The dielectric function of Ag can be decided by Lorentz-Drude model[26-32], see supporting information, S1, S2, S3.

\section{Experimental}

Based on simulations using equations (2) and (3), we select the thickness of every layer considering the fact the $\mathrm{Alq}_{3} / \mathrm{NPB}$ interface is the source of emitting dipole. The device structure is shown in Fig. 2. The refractive index of each layer is also included in this Figure.

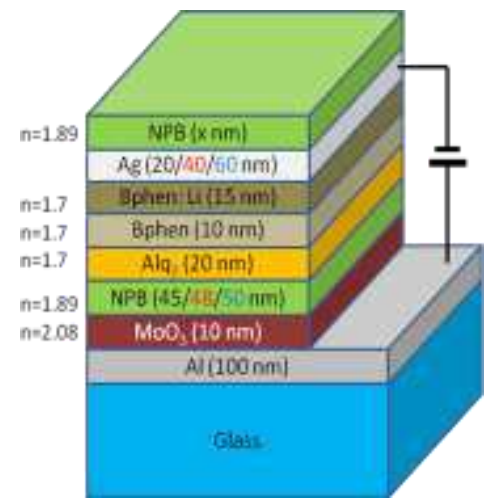

Fig. 2. The structure of green $\mathrm{Alq}_{3}$ TEOLED with NPB capping layer. (Ag cathode and hole transportation NPB layer thicknesses are in one-one correspondence of same color) 
We used $100 \mathrm{~nm} \mathrm{Al}$ as the reflecting anode to fabricate test OLEDs for its excellent smooth film formation properties on glass, which will not induce short circuit. Due to its good conductivity, hole transportation layer NPB thickness was varied to meet the resonance condition (2) and (3) at various phase shift of cathode Ag thickness. Also, we applied NPB as the capping layer material, for it has relative stable property and easy to evaporation.

We fabricated three sets of devices with $\mathrm{Ag}$ cathodes of different thickness $(20 / 40 / 60 \mathrm{~nm})$. The NPB capping layer thickness varied from 0 to $150 \mathrm{~nm}$ with interval of $15 \mathrm{~nm}$. Electroluminescence measurement includes the luminance, $I-V$ curves, current efficiency, luminous power efficiency, and spectra.

\section{Results}

In Fig. 3(a) (c), we can see the Full Width at Half Maximum (FWHM) has a maximum value at capping layer thickness $45 \mathrm{~nm}$. The peak wavelengths do not vary too much at small thicknesses but blue shift occurs when it exceeds 45 nm, see Fig. 3(b).

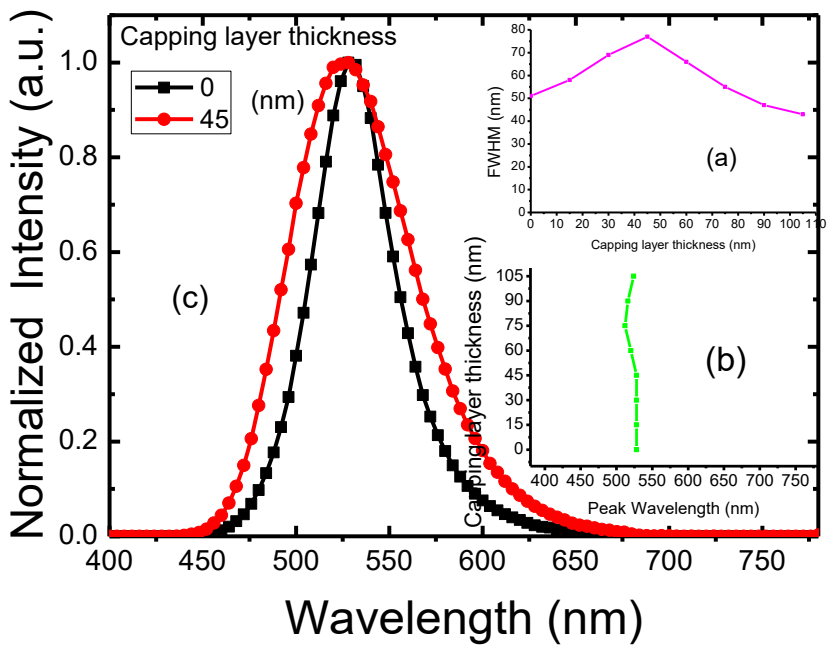

Fig. 3. (a) FWHM vs. capping layer thickness (b) Peak wavelength (c) Normalized spectra of green $\mathrm{Alq}_{3}$ TEOLED

In Fig. 4, we can see the current efficiency also has a maximum value at capping layer thickness $45 \mathrm{~nm}$, and does not change too much at small thicknesses but decreases after $45 \mathrm{~nm}$. In table 1, from $\mathrm{Ag}$ thickness $20 \mathrm{~nm}, 40 \mathrm{~nm}$ and $60 \mathrm{~nm}$, the enhancement ratio of current efficiency varies from $1.05,1.96$, to 3.01 times. The ratio takes on an increasing tendency although the current efficiency decreases.

In Fig. 5, the measured EQE increases with changing the capping layer thickness and reaches a maximum at $45 \mathrm{~nm}$.
Table 1. Current efficiencies at various Ag film thickness and $45 \mathrm{~nm}$ capping layer (green TEOLED of $\mathrm{Alq}_{3}$, @ $180 \mathrm{~mA} / \mathrm{cm}^{2}$ )

\begin{tabular}{cccc}
\hline Cathode Ag thickness (nm) & 20 & 40 & 60 \\
\hline $\begin{array}{c}\text { Current efficiency } \\
\text { (without capping) (cd/A) }\end{array}$ & 6.80 & 2.53 & 0.55 \\
$\begin{array}{c}\text { Current efficiency } \\
\text { (45nm NPB capping) (cd/A) }\end{array}$ & 7.17 & 4.97 & 1.66 \\
Enhancement ratio(times) & 1.05 & 1.96 & 3.01 \\
\hline
\end{tabular}

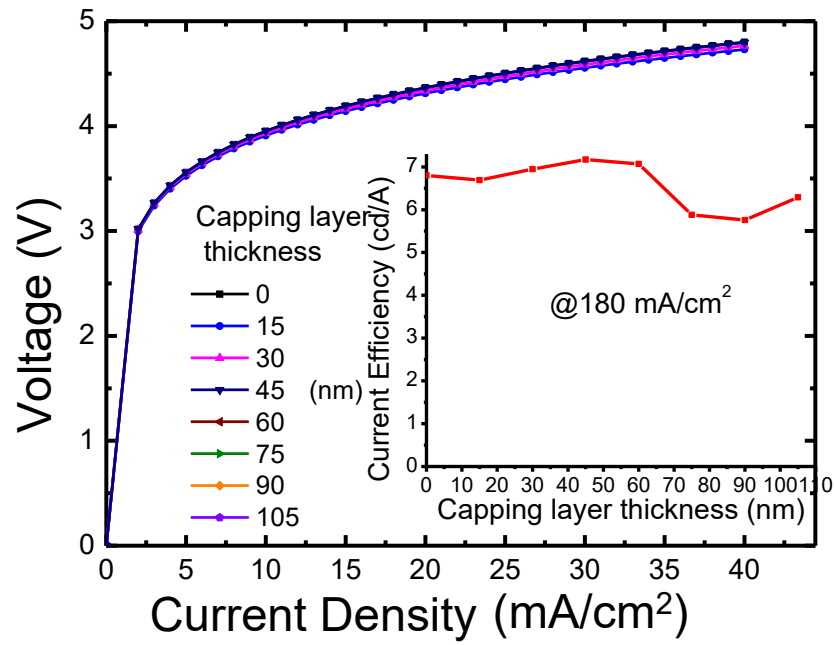

Fig. 4. $J$ - $V$ curves of green $\mathrm{Alq}_{3}$ TEOLED. (@Ag 20nm, the inset Figure is the current efficiency $v s$. capping layer thickness)

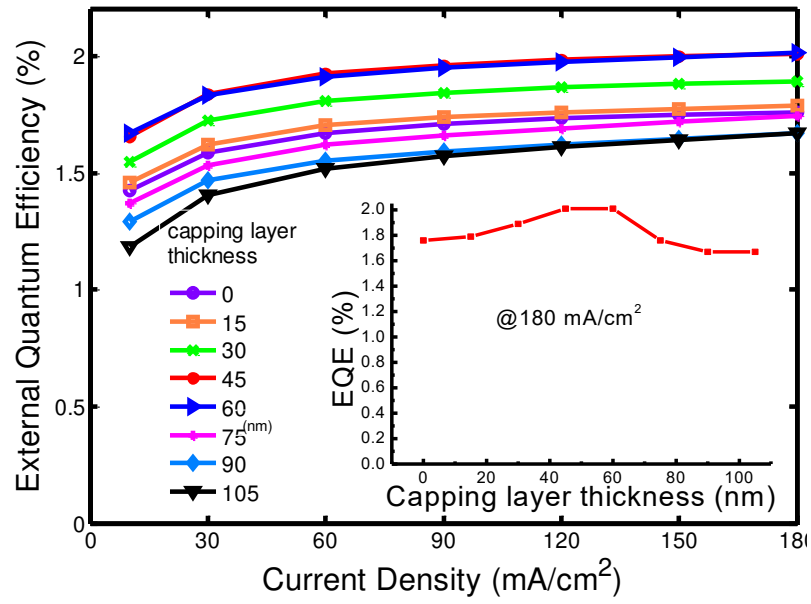

Fig. 5. EQE vs. current density of green TEOLED of $\mathrm{Alq}_{3}$ (The inset Figure is the EQE vs. capping layer thickness)

\section{Discussion}

Next, we discuss the mechanism of enhancing the efficiency with a capping layer. Based on classic cavity theory as given by equation (1), the luminous intensity distribution of the emergent light is decided by two factors. One is the transmittance of the transparent electrode $T_{2}$, and the other is the micro-cavity factor $F(\lambda)$. These two factors are contradictory to the emergent light. Because, based on the basic physics, increased $T_{2}$ will lead to the decreased $R_{2}$ of the $\mathrm{Ag}$ cathode, decreased $R_{2}$ will lead to a 
decreased $F(\lambda)$, then the total gain factor $G(\lambda)$ can't be certain to increasing or decreasing. The high-impact factor plays a decisive role.

From the real experiments results, we can see, the current efficiencies, EQEs, FWHMs increase with increasing the capping layer thickness before $45 \mathrm{~nm}$, i.e., the $G(\lambda)$ increases. As we know, the FWHM is an indicator of the strength of micro-cavity effect[2], the greater FWHM, the weaker $F(\lambda)$, the smaller $R_{2}$ and the greater $T_{2}$. Can we determine that $T_{2}$ is the decisive factor? No. It is necessary to calculate it more accurately.

\section{Transmittance of Ag film Cathode with a Capping Layer}

We first simulate the transmittance of $\mathrm{Ag}$ cathode with different NPB capping layer thicknesses as shown in Fig. 6(a).

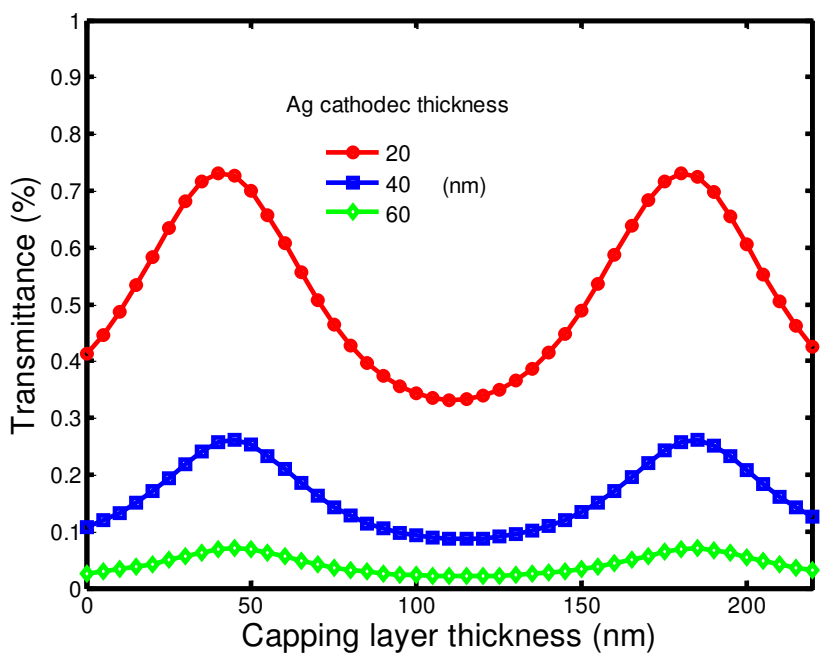

(a)

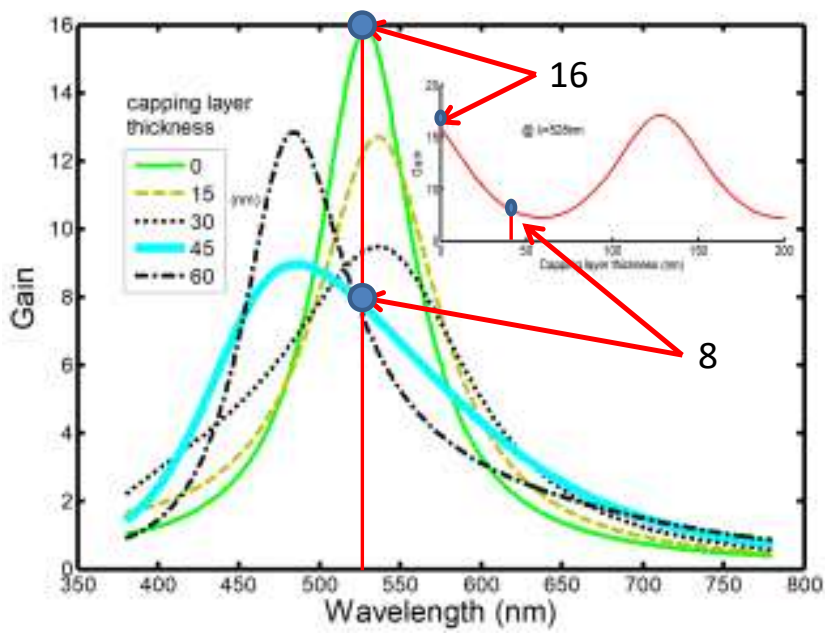

(b)

Fig. 6. (a) The transmittance of $20,40,60 \mathrm{~nm}$ Ag $v$ s. NPB capping layer thickness @ $\lambda=528 \mathrm{~nm}$. (b) $\mathrm{G}(\lambda) v s$. wavelength. (20nm Ag cathode and 100nm Al reflecting anode, different capping layer thickness; the inset Figure is the gain factor $v s$. capping layer thickness @ $\lambda=528 \mathrm{~nm})$

For the periods of optical path length parameters, in the case of taking no account of dielectric loss, the transmittance is also periodic. At $45 \mathrm{~nm}$ thickness of capping layer, the transmittance reaches the maximum value of $73 \%$ (40\%, without capping layer). This fully complies with the metal induced transmission (MIT) effect. The maximum efficiency value of the green color OLED is consistent with the maximum transmittance of the $\mathrm{Ag}$ cathode superficially.

Gain factor of the micro-cavity $G(\lambda)=F(\lambda) \cdot T_{2}$

Fig. 6(b) provides the calculated gain factors $G(\lambda)$ according to the equation (1). We can find that although the maximum transmittance is at $45 \mathrm{~nm}$ thick capping layer, the gain factor $G(\lambda)$ is only half of that without capping layer, see the dots pointed by the arrows in the figure. The reason is just as described before 5.1 section and it seems the $F(\lambda)$ is the decisive factor. So, the simulation results and real tested results go against each other.

In fact, our measured result is that current efficiency and quantum efficiency all increased compared with bare Ag cathode film, as shown in table 1 and Fig. (4) (5). Especially, the $60 \mathrm{~nm}$ silver cathode with $45 \mathrm{~nm}$ NPB capping layer nearly triples the current efficiency. There must be other factors to induce this result.

In the above discussed film optical simulations, we only consider the optical interference effect in normal direction. In the situations of large angle incidence, the dipole radiation interacting with metal electrodes will produce the SPPs [33] which greatly impact the EQE of the OLED due to the exciton's near-field radiation characteristics.

\section{SPP (Surface Plasmon Polaritons)}

According to classic optical theory, when the incident angle is greater than the critical angle of total reflection, an evanescent wave will appear. When the evanescent wave interacts with the thin metal film surface and match the wave-vector or momentum condition, surface plasmon loss will occur. SPPs are a trapped electromagnetic surface mode at the interface between a metal and a dielectric[34]. They are a combined collective oscillation of electromagnetic field and the surface charges of the metal, which can be activated by transverse magnetic (TM) wave. The electromagnetic field of SPPs at dielectric-metal interface can be 
obtained from the solution of Maxwell's equations in each medium and the associated boundary conditions, and the dispersion relation is illustrated in Fig. 7[33]. Where, $\varepsilon_{\mathrm{m}}$ is the dielectric function of the metal, $\varepsilon_{\mathrm{d}}$ that of the adjacent dielectric. Researchers have demonstrated that, due to the loss property of metals, the amplitude of SPPs decays exponentially with increasing distance into each medium from the interface. They can only propagate along the direction of the interface, so it is a kind of guided wave mode, causing non-radiative loss.

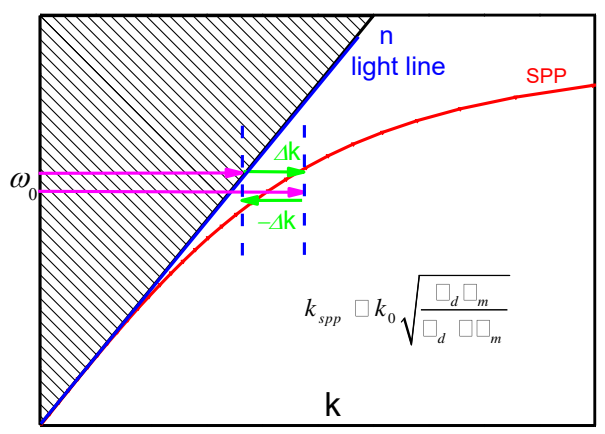

Fig. 7. The dispersion relation of SPPs

In Fig. $7, k_{\mathrm{Spp}}>k_{0}$, commonly, due to the mismatch of the wave-vector, the far-field light cannot activate the SPPs directly, but we still can add wave-vector $\Delta k$ to activate it by some special means such as Kretschmann method, Otto method, etc[34], which is based on photon tunneling effect. The periodic structure and roughness on metal surface can excite SPPs directly because of the diffraction effect, and vice versa SPPs can be recovered to light through all these methods by reduced wave-vector $-\Delta k$ [34].

Another means to activate SPP is the near-field method. The near-field excited SPPs widely exist in the OLEDs, an essential factor which leads to the low quantum efficiency of OLEDs.

\section{Role of SPPs in OLED}

In OLEDs, since the near-field radiation field can be expressed as a sum of plane waves and each plane wave has its in-plane wave-vector component $k_{\mathrm{x}}$, it can be coupled into SPPs on a near metal surface directly when the momentum condition is matched. Power lost to SPPs can account for up to $40 \%$ of the total power[35] and it is essential to explore whether some of the power lost to SPP modes can be recovered, thereby improving device efficiency.

A classical model [36-39], the power dissipated of a dipole is also a function of $k_{\mathrm{x}}$, is called the power dissipation spectrum of wavelength (PDS). From it, we can gain insight into the nature of the modes that are present and the strength of power coupled to them. The out-coupling efficiency is obtained by integrating the power of a certain range compared to the total integrated power.

PDS aims at a specific wavelength and power dissipation map (PDM) focuses on all wavelengths. We use color depth to represent the degree of the energy loss. In fact, the profile of PDM just comprises the dispersion relation with more information.

In Fig. 8(a), we provide a PDS of a BEOLED via numerical

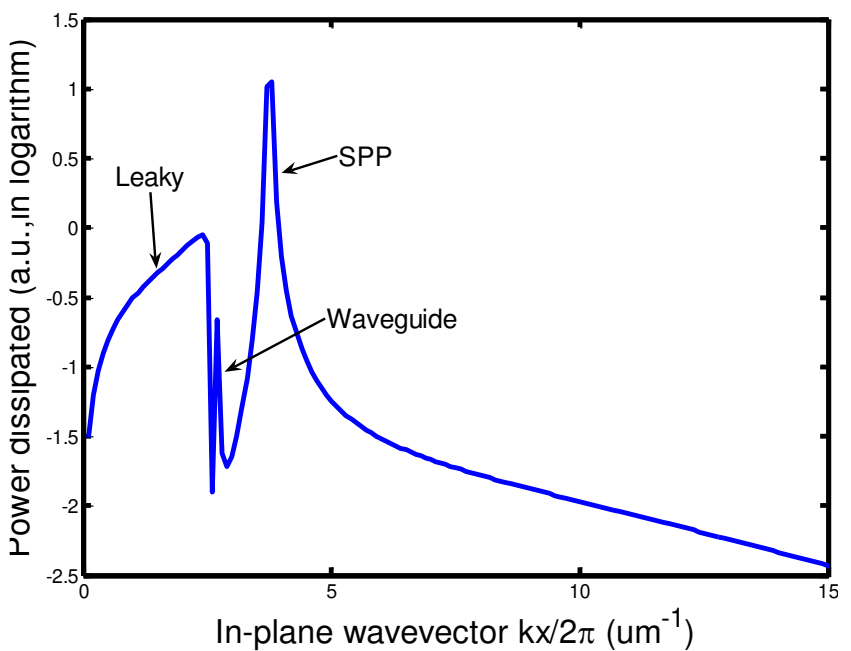

(a)

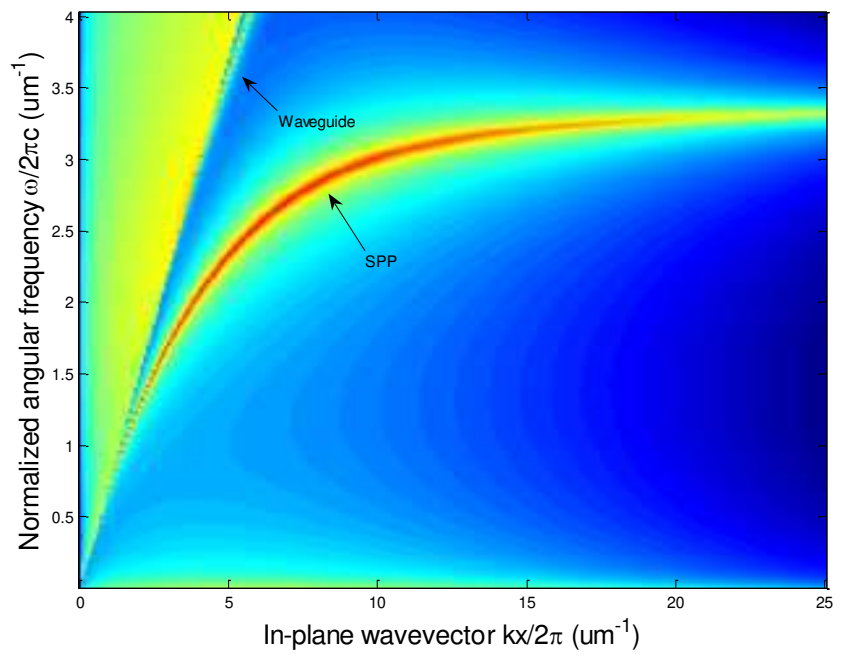

(b)

Fig. 8. (a) PDS of BEOLED with Ag reflecting cathode @ $\lambda=528 \mathrm{~nm}$. (b) PDM of BEOLED with Ag reflecting cathode.

simulations. The leaky mode can couple out from emitter and contributes the main part of the quantum efficiency. However the waveguide and SPP modes are lossy modes. As shown in Fig. 8(b), from blue to red, it demonstrates the degree of deepened energy losses (in logarithm), see supporting information, S4, S5. 
In normal BEOLED devices, SPPs occur on the reflecting cathode, and in TEOLED, SPPs occur on the reflecting anode and semi-transparent metal cathode. Therefore, to recover SPPs loss in TEOLED is especially significant.

When the metal film is thinner than the decay depth of the SPP mode, the emitter can activate SPP onto both sides of the cathode, named as SPP2 (bottom) and SPP1 (top), and they will interact each other to give rise to coupled modes under various upper capping layer (DMD). When the capping layer reaches a certain thickness, the coupling level gets to the top. Many PDS are provided in pseudo-3D Fig. 9(a), showing varied coupling level between SPP1 and SPP2.

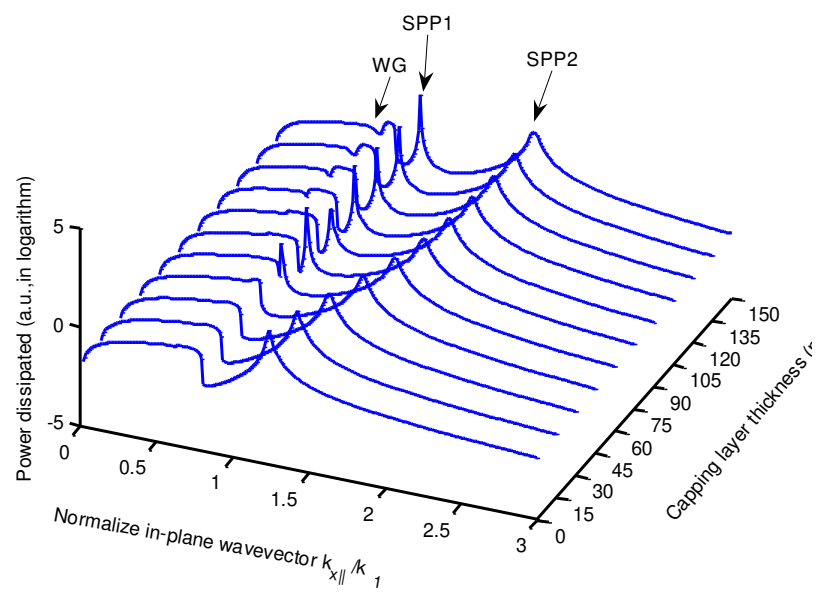

(a)

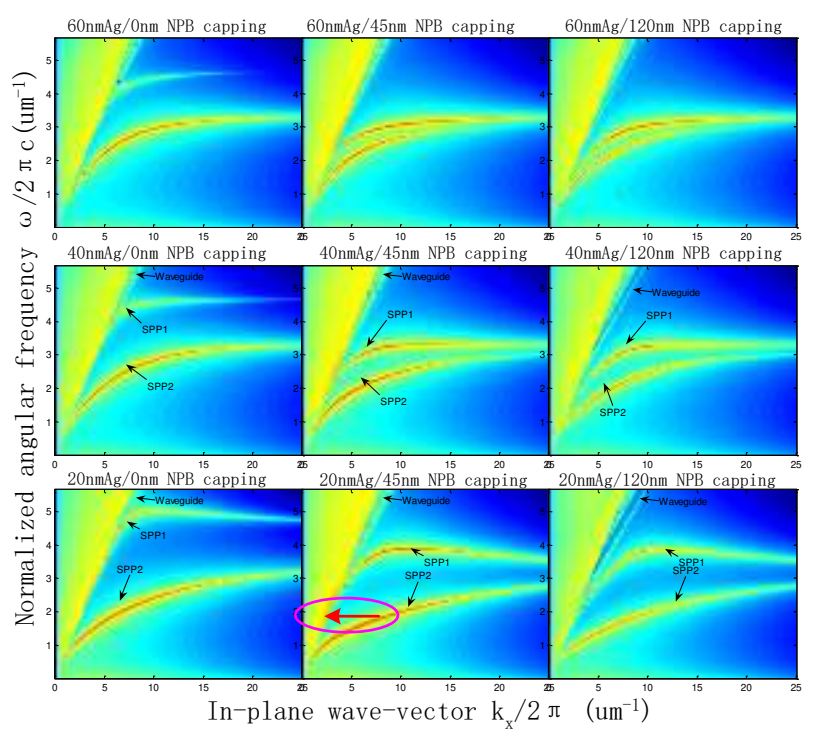

(b)

Fig. 9. (a) PDS of Top-emitting OLED of 20nm Ag cathode with different capping layer thickness @ $\lambda=528 \mathrm{~nm}$. (b) PDM of Top-emitting OLED with different $\mathrm{Ag}$ cathode and NPB capping layer thickness.

Without capping layer, SPP1 is approximately 2 orders of magnitude lower than that of SPP2, which is hardly visible. Above $45 \mathrm{~nm}$ thick capping layer, SPP2 are strongly coupled to SPP1, see Fig. (10). Based on the reciprocity principle of radiation transfer, SPP1 can be easily recovered to light under the moment match condition, vice versa.

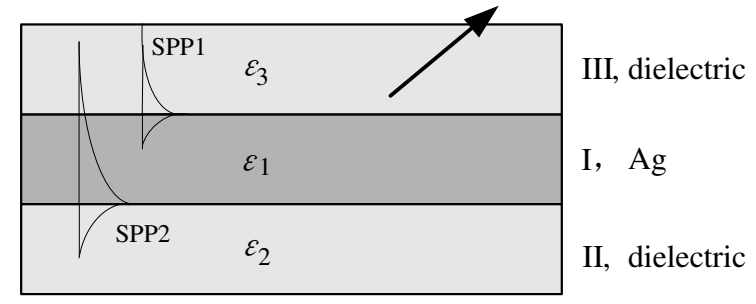

Fig. 10. Coupled SPPs on the D/M/D structure Ag cathode.

More in-depth research reveals that, strictly, it is incorrect to refer to the SPP modes as coupling one to another respectively; rather they are in an integration of coupled SPPs on both sides.

Fig. 9(b) illustrates nine PDMs of different Ag film cathode and capping layer thicknesses. We can see that the coupling level is determined by the $\mathrm{Ag}$ film thickness and the capping layer thickness. On the bottom middle inset image, near $528 \mathrm{~nm}$ (normalized angular frequency, $1.89 \mathrm{um}^{-1}$ ), we can see the coupling level is the highest for both the color of the two curves are the deepest (circled in the Figure).

\section{Conclusions}

We use the transfer matrix method in film optical calculation to optimize the metal electrodes' parameters of the TEOLED. Based on the principle of the micro-cavity effect, the optical path parameters are also optimized for the thicknesses of different functional organic films. We found that the quantum efficiencies are enhanced compared to that with bare Ag cathode in fabricated fluorescent $\mathrm{Alq}_{3}$ green TEOLED with different thickness of NPB capping layer on Ag cathode film. The highest EQE is at $45 \mathrm{~nm}$ capping layer. But it cannot be fully explained by classic film optics theory. Incorporation with the near-field radiation of electric dipole theory, based on PDS and PDM, via the numerical simulations, we explain that the enhancements of efficiencies arise from 3 factors: the increased transmittance of the cathode $\mathrm{Ag}$ film, the varied gain of the micro-cavity, the dominant coupled SPPs.

Such devices with a dielectric capping layer (DMD) increase the optical outcoupling and the efficiencies of TEOLEDs with 
very simple means, which is very significant for the low-cost commericialization application in display area.

\section{Author Contribution}

Min Qian, Dong-Ying Zhou: Conceptualization, Methodology, Funding acquisition, Project administration.

Xue-Liang Wang: Experimental, Investigation, Formal analysis, Visualization, Software, Writing original draft.

Funding This work was supported by the Natural Science Foundation of the Jiangsu Higher Education Institutions of China (19KJD510006)

Availability of Data and Material The authors confirm that the data and material supporting the findings of this study are available within the article and suporting informantion.

Code Availability The authors confirm that the code of this study are available within the suporting informantion.

\section{Declarations}

Ethics Approval Not applicable.

Consent to Participate Not applicable.

Consent for Publication All authors have given their consent for the publication of this article in the Plasmonic Journal.

Conflict of Interest The authors declare no competing interests.

\section{References}

1 L.J.R. Dodabalapur, T. M. Miller, and E. W. Kwock (1994) Microcavity effects in organic semiconductors. Appl Phys Lett 64(19). https://doi.org/10.1063/1.111606

2 L.J.R. Dodabalapur, R. H. Jordan, T. M. Miller, R. E. Slusher, and Julia M. Phillipse (1996) Physics and applications of organic microcavity light emitting diod. J Appl Phys. https://doi.org/10.1063/1.363768

3 L.Z. Wenyu Ji, Tianyu Zhang, Guoqiang Liu, Wenfa Xie, Shiyong Liu, Hanzhuang Zhang, Liying Zhang, and Bin Li (2009) Top-emitting white organic light-emitting devices with a one-dimensional metallic-dielectric photonic crystal anode. Opt Lett 34(18):2703. https://doi.org/10.1364/OL.34.002703
4 S. Tokito, T. Tsutsui, Y. Taga (1999) Microcavity organic light-emitting diodes for strongly directed pure red, green, and blue emissions. J Appl Phys 86(5):2407. https://doi.org/10.1063/1.371068

5 J. Tischler, M. Bradley, V. Bulović, J. Song, A. Nurmikko (2005) Strong Coupling in a Microcavity LED. Phys Rev Lett 95(3) https://doi.org/10.1103/PhysRevLett.95.036401

6 Y.-I.P. Chang-Jun Lee, Jang-Hyuk Kwon, $\dagger$ and Jong-Wook Park (2005) Microcavity effect of top-emission organic light-emitting diodes using Aluminum cathode and anode. bull korea chem soc. https://doi.org/10.5012/bkcs.2005.26.9.1344

7 G. Gustafsson, Y. Cao, G. Treacy, F. Klavetter, N. Colaneri, A. Heeger (1992) Flexible light-emitting diodes made from soluble conducting polymers Nature 357(6378):477-479. https://doi.org/10.1038/357477a0

8 W.S. Wong, A. Salleo, Flexible electronics: materials and applications, Springer2009.

9 S. Chen, Y. Zhao, G. Cheng, J. Li, C. Liu, Z. Zhao, Z. Jie, S. Liu (2006) Improved light outcoupling for phosphorescent top-emitting organic light-emitting devices. Appl Phys Lett 88(15):153517. https://doi.org/10.1063/1.2190274

10 H. Riel, S. Karg, T. Beierlein, B. Ruhstaller, W. Rieß (2003) Phosphorescent top-emitting organic light-emitting devices with improved light outcoupling Appl Phys Lett 82(3):466. https://doi.org/10.1063/1.1537052

11 J. Lee, S. Hofmann, M. Furno, M. Thomschke, Y.H. Kim, B. Lüssem, K. Leo (2011) Influence of organic capping layers on the performance of transparent organic light-emitting diodes. Opt Lett 36(8):1443-1445 https://doi.org/10.1364/OL.36.001443

12 H. Riel, S. Karg, T. Beierlein, W. Rieß, K. Neyts (2003) Tuning the emission characteristics of top-emitting organic light-emitting devices by means of a dielectric capping layer: An experimental and theoretical study. J Appl Phys 94(8):5290-5296. https://doi.org/10.1063/1.1605256

13 Q. Huang, K. Walzer, M. Pfeiffer, K. Leo, M. Hofmann, T. Stübinger (2006) Performance improvement of top-emitting organic light-emitting diodes by an organic capping layer: An experimental study. J Appl Phys 100(6):064507. https://doi.org/10.1063/1.2338145

14 G.Z. Ran, W.Q. Zhao, L, G, L. Dai, G.G. Qin (2006) Role of the dielectric capping layer in enhancement of light outcoupling for semitransparent metal-cathode organic light-emitting devices. J Optics A 8(9):733. https://doi.org/10.1088/1464-4258/8/9/004

15 T. Chen, D. Ghosh, D. Krautz, S. Cheylan, V. Pruneri (2011) Highly stable Al-doped $\mathrm{ZnO}$ transparent conductors using an oxidized ultrathin metal capping layer at its percolation thickness. Appl Phys Lett 99(9):181. https://doi.org/10.1063/1.3631674

16 J.W. Huh, J. Moon, J.W. Lee, D.-H. Cho, J.-W. Shin, J.-H. Han, J. Hwang, 
C.W. Joo, H.Y. Chu, J.-I. Lee (2012) Directed emissive high efficient white transparent organic light emitting diodes with double layered capping layers. Org Electron 13(8):1386-1391. https://doi.org/10.1016/j.orgel.2012.04.005

17 Q. Huang, K. Walzer, M. Pfeiffer, K. Leo, M. Hofmann, T. Stuebinger (2006) Performance improvement of top-emitting organic light-emitting diodes by an organic capping layer: An experimental study. J Appl Phys 100(6). https://doi.org/10.1063/1.2338145

18 K. Hong, K. Kim, S. Kim, I. Lee, H. Cho, S. Yoo, H.W. Choi, N.-Y. Lee, Y.-H. Tak, J.-L. Lee (2011) Optical Properties of WO3/Ag/WO3Multilayer As Transparent Cathode in Top-Emitting Organic Light Emitting Diodes. J Phys Chem C 115(8):3453-3459. https://doi.org/10.1021/jp109943b

19 H.A. Macleod, Thin-Film Optical Filters, CRC Press2017.

20 M. Qian, X.-B. Shi, J. Ma, J. Liang, Y. Liu, Z.-K. Wang, L.-S. Liao (2015) A stacked $\mathrm{Al} / \mathrm{Ag}$ anode for short circuit protection in ITO free top-emitting organic light-emitting diodes. RSC Advances 5(117):96478-96482. https://doi.org/10.1039/C5RA18132A

21 M. Qian, Q. Liu, W.-S. Li, J.-X. Man, Y.-B. Zhao, Z.-H. Lu (2018) Dual Ag electrodes for semitransparent organic light-emitting diodes. Org Electron 57(98-103. https://doi.org/10.1016/j.orgel.2018.02.028

22 M.A. Karim, A.A. Awwal, Optical computing: an introduction, 1992.

23 R.R. Willey, Practical design and production of optical thin films, 2002.

24 A. Chen, L. Ge, Y. Dai, L. Shi, X. Liu, D. Han, J. Zi, Transfer Matrix Method for Optical Calculations of Multilayered Topological Insulators, 2017.

25 K.F. Warnick, Numerical Methods for Engineering: An Introduction Using MATLAB and Computational Electromagnetics Examples, SciTech Pub.2011.

26 J.H. Weaver, C. Krafka, D. Lynch, E. Koch (1981) Optical properties of metals. Appl Opt 20(7). https://doi.org/10.1364/AO.20.1124_1

27 D. Nash, J. Sambles (1996) Surface plasmon-polariton study of the optical dielectric function of silver. J Mod Optic 43(1):81-91. https://doi.org/10.1080/09500349608232725

28 E.J. Prucha, E.D. Palik, Handbook of optical constants of solids, vol. 1, 2, and 3, 1998.

29 A.D. Rakić, A.B. Djurišić, J.M. Elazar, M.L. Majewski (1998) Optical properties of metallic films for vertical-cavity optoelectronic devices. Appl Opt 37(22):5271. https://doi.org/10.1364/AO.37.005271

30 J. Shibayama, R. Ando, A. Nomura, J. Yamauchi, H. Nakano (2009) Simple trapezoidal recursive convolution technique for the frequency-dependent FDTD analysis of a Drude-Lorentz model. IEEE Photonics Technology Letters 21(2):100-102. https://doi.org/10.1109/LPT.2008.2009003

31 M. Kadi, A. Smaali, R. Outemzabet (2012) Analysis of optical and related properties of tin oxide thin films determined by Drude-Lorentz model. Surf
Coat Technol 211(45-49. https://doi.org/10.1016/j.surfcoat.2011.10.027

32 R.L. Olmon, B. Slovick, T.W. Johnson, D. Shelton, S.-H. Oh, G.D. Boreman, M.B. Raschke (2012) Optical dielectric function of gold. Physical Review B 86(23):235147. https://doi.org/10.1103/PhysRevB.86.235147

33 W.L. Barnes, A. Dereux, T.W. Ebbesen (2003) Surface plasmon $\begin{array}{lll}\text { subwavelength } \quad \text { optics. } & \text { Nature }\end{array}$ https://doi.org/10.1038/nature01937

34 H. Raether, Surface plasmons on smooth surfaces, Springer1988.

35 L. Smith, J. Wasey, W.L. Barnes (2004) Light outcoupling efficiency of top-emitting organic light-emitting diodes. Appl Phys Lett 84(16):2986-2988. https://doi.org/10.1063/1.1712036

36 W. Barnes (1999) Electromagnetic crystals for surface plasmon polaritons and the extraction of light from emissive devices. J Lightwave Technol 17(11):2170. https://doi.org/10.1109/50.803008

37 R. Chance, A. Prock, R. Silbey, Molecular fluorescence and energy transfer near interfaces, 1978.

38 G.W. Ford, W.H. Weber (1984) Electromagnetic interactions of molecules with metal surfaces. Phys Rep 113(4):195-287. https://doi.org/10.1016/0370-1573(84)90098-X

39 P. Hobson, J. Wasey, I. Sage, W. Barnes (2002) The role of surface plasmons in organic light-emitting diodes. IEEE J Sel Top Quant 8(2):378-386. https://doi.org/10.1109/2944.999193 